\title{
JET radiative mantle experiments in ELMy H-Mode
}

\author{
J D Strachan $\dagger$, R Budny $\dagger$, I Coffey $\ddagger$, P Dumortier§, C Grisolia\|, P Harbour $\uparrow$, \\ M von Hellermann $\uparrow$, D Hillis ${ }^{+}$, T Hoang $\|, \mathrm{J} \mathrm{Hogan}^{+}$, L D Horton $\uparrow$,

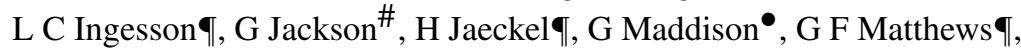 \\ M Mayer ${ }^{\circ}$, A Messiaen§, P Monier-Garbet\|, M F N Nave ${ }^{\diamond}$, J Ongena§, \\ $\mathrm{J} \mathrm{Rapp}^{\circ}$, B Unterberg ${ }^{\circ}, \mathrm{M}$ Wade $^{+}$and K-D Zastrow 9 \\ JET Joint Undertaking, Abingdon, Oxon, OX14 3EA, UK \\ $\dagger$ PPPL, Princeton University, USA \\ \$ Queens University, Belfast, UK \\ $\S$ KMS/ERM, Belgium \\ $\|$ CEA Cadarache, France \\ I JET Joint Undertaking, UK \\ + ORNL, Oak Ridge, USA \\ \# General Atomics, San Diego, USA \\ - EURATOM/UKAEA Fusion Association, Culham, UK \\ - Institut fuer Plasmaphysik, Forchungszentrum Juelich GmbH, Germany \\ $\diamond$ Associacao EURATOM/IST, CFN, Lisbon, Portugal
}

Received 29 September 1999

\begin{abstract}
Radiative mantle experiments were performed on JET ELMy H-mode plasmas. The septum configuration was used where the $X$-point is embedded into the top of the septum. Argon radiated $50 \%$ of the input power from the bulk plasma while $Z_{\text {eff }}$ rose from an intrinsic level of 1.5 to about 1.7 due to the injected argon. The total energy content and global energy confinement time decreased $15 \%$ when the impurities were introduced. In contrast, the effective thermal diffusivity in the core confinement region $(r / a=0.4-0.8)$ decreased by $30 \%$. Usually, JET ELMy H-mode plasmas have confinement that is correlated to the edge pedestal pressure. The radiation lowered the edge pedestal and consequently lowered the global confinement. Thus the global confinement was changed by a competition between the edge pedestal reduction lowering the confinement and the weaker RI effect reducing the core transport coefficients. The ELM frequency increased from $10 \mathrm{~Hz}$ type-I ELMs, to $200 \mathrm{~Hz}$ type-III ELMs. The energy lost by each ELM reduced to $0.5 \%$ of the plasma energy content.
\end{abstract}

Highly radiating plasmas occur when high- $Z$ impurities such as neon, argon, nitrogen, and krypton are injected into tokamak plasmas. The consequential reduction of power flowing to the first wall components has potential for improving reactor power handling [1]. However, experiments feature different consequences to the plasma energy confinement [2]. On the one hand, impurity injection into TEXTOR [3], ISX-B [4], DIII-D [5], and AUG [6,7] improved confinement, while on the other hand, impurity injection into JET [8] degraded confinement. These experiments feature many differences, (such as the use of limiters on TEXTOR and ISX-B and divertors on the other machines) which could be playing some role in the different experimental results obtained.

This paper reports results from JET impurity seeding in ELMy H-mode plasmas. Our philosophy was to have a joint experimental programme including many experimentalists with 
experience of radiative mantle experiments. Our strategy was to use their experience to improve the JET ELMy H-Mode, since that regime is a reactor-relevant regime that could benefit from reduced power flows to the divertor. Our intention was to perform the experiment in a similar manner as TEXTOR (albeit in a different plasma regime and with a different plasma boundary) and thus assess the origin of the different results.

The experiment was limited to a two-day duration, and so cannot be considered to be optimized. In particular, the upper limit of the radiative fraction was probably not obtained. However, plasmas were obtained which followed the techniques used on TEXTOR and DIII-D. We observed:

(i) Confinement degradation similar to that seen previously on JET [8], although with higher densities than previously achieved.

(ii) The confinement degradation correlated with the reduction in the edge pedestal pressure as seen commonly on JET [9].

(iii) The impurity seeding reduced transport at $r / a=0.4-0.8$ similar to TEXTOR [3].

(iv) The radiation fraction was high, and the energy lost per ELM was substantially reduced.

(v) Even during the impurity seeding, the volume averaged $Z_{\text {eff }}<2$.

28 discharges (ELMy H-Modes at 2.5 MA and $2.5 \mathrm{~T}$ heated by $12 \mathrm{MW}$ neutral beams) with argon or neon radiating mantles were performed. The argon produced about twice as much core radiation as the neon. Two divertor configurations were tried: (1) the corner configuration (with the strike points at the cryo-pump port location) should maximize the divertor's impurity pumping capability and (2) the septum configuration with the $X$-point embedded into the septum top. This configuration is like a pump limiter, since the plasma is bounded by a material surface but the SOL has access to the divertor pumps. JET septum operation features an H-mode power threshold 2-3 times lower than other divertor configurations. Thus septum operation has the conceptual (but not yet proven) advantage of allowing more radiation without reducing the power flow through the $X$-point to below the H-mode power threshold.

The bulk of the experimental campaign was devoted to modification of the deuterium and argon gas injection rates in septum plasmas. The best results came with $3 \times 10^{22}$ electrons s $^{-1}$ from $\mathrm{D}_{2}$ gas puffing, and a step-down evolution of argon gas (figures 1 and 2). The radiating plasma is in quasi-equilibrium with stationary parameters lasting about 5 energy confinement times (figure 2). The difference between the present campaign, and previous JET highly radiating plasma experiments [8] is primarily that higher deuterium gas fuelling was used in conjunction with the argon puffing (figure 1), and that the septum configuration was explored.

Both TEXTOR [10] and DIII-D publications describe two additional factors important for confinement enhancement during radiative mantle experiments, namely (1) avoiding MARFE formation and (2) the plasma edge distance to the outer wall. In these JET experiments, variations of the position of the outer edge did not affect confinement and MARFEs did not occur in any of these plasmas.

The input power was about $12 \mathrm{MW}$ from 17 to $23 \mathrm{~s}$ in figure 1, and the radiated power rose approximately from $1 \mathrm{MW}$ bulk and $2 \mathrm{MW} X$-point radiation to $5 \mathrm{MW}$ bulk and $3 \mathrm{MW}$ $X$-point radiation about $1.2 \mathrm{~s}$ after the argon puffing began. The $X$-point radiation stayed about constant although less spiky as the ELM behaviour changed in the presence of the higher mantle radiation (figure 2). The ELMs had been regular type-I ELMs of about $10 \mathrm{~Hz}$ frequency without argon and became type-III ELMs ( $200 \mathrm{~Hz}$ frequency). Due to the high density, $Z_{\text {eff }}$ rose only modestly from about 1.5 without argon to 1.7 with the argon (figure 2 ). The central density rose about $10 \%$ to about $8.5 \times 10^{19} \mathrm{~cm}^{-3}$ with the argon but the central electron and ion temperature fell from about $3 \mathrm{keV}$ to about $2.5 \mathrm{keV}$. With the lower temperature, the $\mathrm{d}(\mathrm{d}, \mathrm{n}) 3 \mathrm{He}$ neutron emission fell from 3.5 to $2.5 \times 10^{15} \mathrm{~s}^{-1}$. The global (magnetic) confinement time 


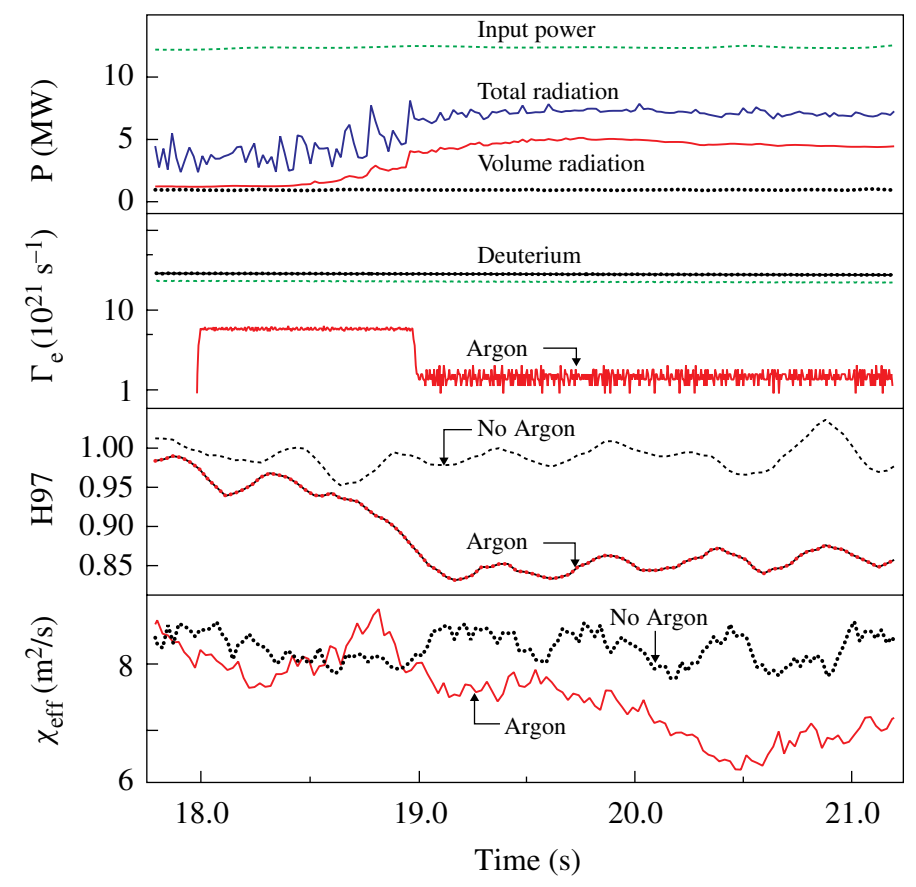

Figure 1. The time evolution of the argon radiative mantle plasma 46980 (solid curves) and the noargon reference plasma 46978 (dashed curves). From top to bottom: the input power was $12 \mathrm{MW}$, the volume radiation from the reference plasma was $1 \mathrm{MW}$, the argon caused the volume radiation to rise to $5 \mathrm{MW}$, while the $X$-point radiation remained at $2 \mathrm{MW}$; the total electron injection rate from deuterium (the reference plasma had slightly less deuterium fuelling) and from argon; H97; the TRANSP calculated effective conductivity coefficient at $R=3.5 \mathrm{~m}$.

fell $15 \%$, although the TRANSP deduced effective conductivity coefficient at $R=3.5 \mathrm{~m}$ fell about $30 \%$ (figure 1).

The bolometer signals [11] were Abel unfolded to determine the radiation profile (figure 3) of the top half of the poloidal cross section. The $X$-point radiation dominates the emission in the bottom half and obscures the core radiation pattern. The argon impurity enhanced the radiation across the entire profile. The JET argon bulk radiation profiles were similar to TEXTOR neon profiles [10] although the JET radiation power density is about half that in TEXTOR. Both plasmas were at $85 \%$ of the Greenwald density limit, with similar beam heating power densities. The width of the JET edge radiation mantle is about half the TEXTOR neon mantle width, or one-quarter the TEXTOR argon width.

The fuel depletion by the seeded impurity was acceptably small $\left(n_{\mathrm{d}} / n_{\mathrm{e}}=0.8\right)$ for JET as for TEXTOR (figure 2). Since the JET plasmas had large deuterium fuelling, the higher density caused high radiation levels at modest impurity injection rates (figure 1). By contrast, TEXTOR usually turned off the gas fuelling during the impurity injection and relied upon the enhanced confinement to reach high density. Charge exchange spectroscopic observation of carbon densities indicated that the intrinsic impurity levels were not affected by the radiation mantle, and remained constant.

The argon radiation mantle discharges achieved good confinement when compared with other JET plasmas taken at the same plasma conditions (figure 4). The comparison data in figure 4 span the earlier JET divertor configurations, comprising all the data in the official 


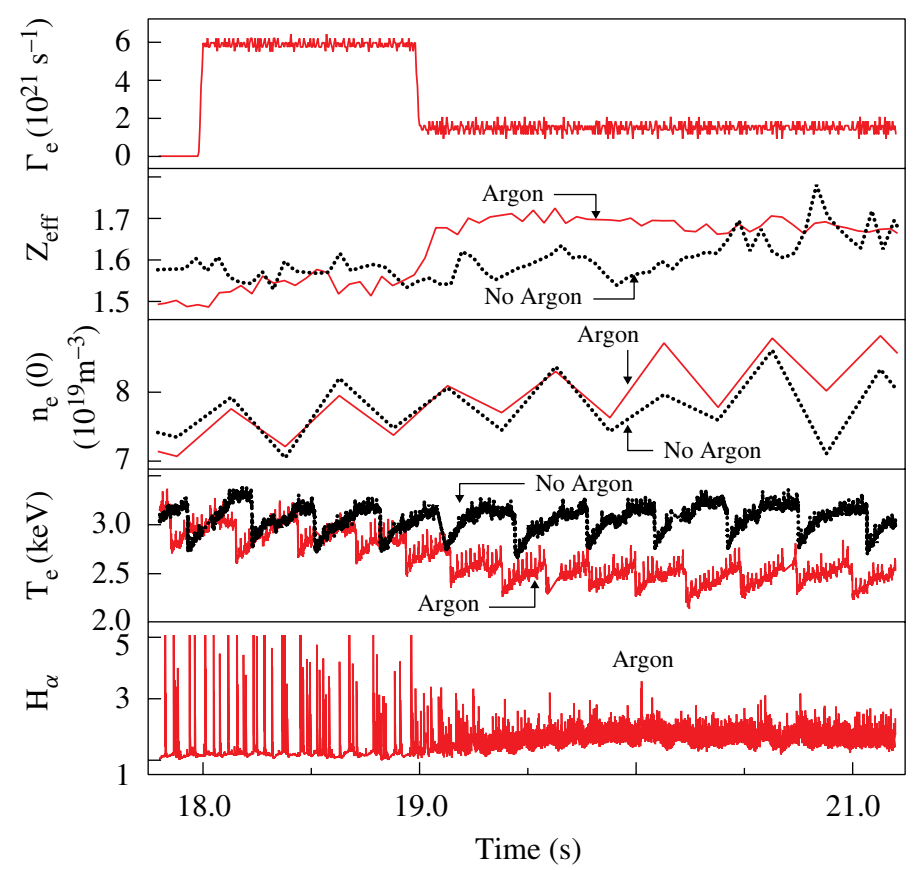

Figure 2. The time evolution of the argon (46980) and reference plasma (46978) showing, fom top to bottom: the electron injection rate due to the argon gas fuelling; the average $Z_{\text {eff }}$ measured by visible bremsstrahlung; the Thomson scattering deduced central density; the ECE measured central electron temperature; and the $H_{\alpha}$ light.

JET steady-state data bases having the quoted machine settings. The densities are $20 \%$ higher than have been otherwise achieved. Apparently, the radiative mantle was useful to achieve higher density as on TEXTOR and DIII-D but at the expense of a degraded confinement unlike TEXTOR and DIII-D. The reference plasma (no-argon, septum configuration) also had slightly higher density than the other divertor plasmas (at these conditions). Possibly, the density is higher, in part, due to septum operation as well as due to the radiation mantle.

The energy balance was analysed using the TRANSP code. The diagnostic consistency (comparison of the kinetic and magnetic energy contents) was not particularly good, with the magnetic energy exceeding the kinetic energy content by about $25 \%$, for both the no-argon reference case and the selected argon radiative mantle plasma. The coupling between the electrons and the ions was large due to the low electron temperatures and the high densities, so that separate electron and ion energy balances could not be distinguished. An effective $\chi$ (total conducted power loss described by a transport coefficient governed by the average of the electron and ion temperature gradient) was reduced by about 30\% (figures 1 and 5) which is a similar reduction as was reported on TEXTOR [3] in this region of the plasma (figure 5). The reduction exists in the calculated energy balance primarily because of the reduced conducted power flow through the core region due to the radiated power in figure 3 . The previously mentioned difficulties inverting the radiation profiles also make the conduction coefficient uncertain in a magnitude that has yet to be determined.

The magnitude of the effective $\chi$ is about $0.5 \mathrm{~m}^{2} \mathrm{~s}^{-1}$ in the core confinement region (figure 5). The analysis of the time evolution of the soft $\mathrm{x}$-ray emission indicates similar impurity particle transport in that spatial location (figure 5). However, the analysis of 


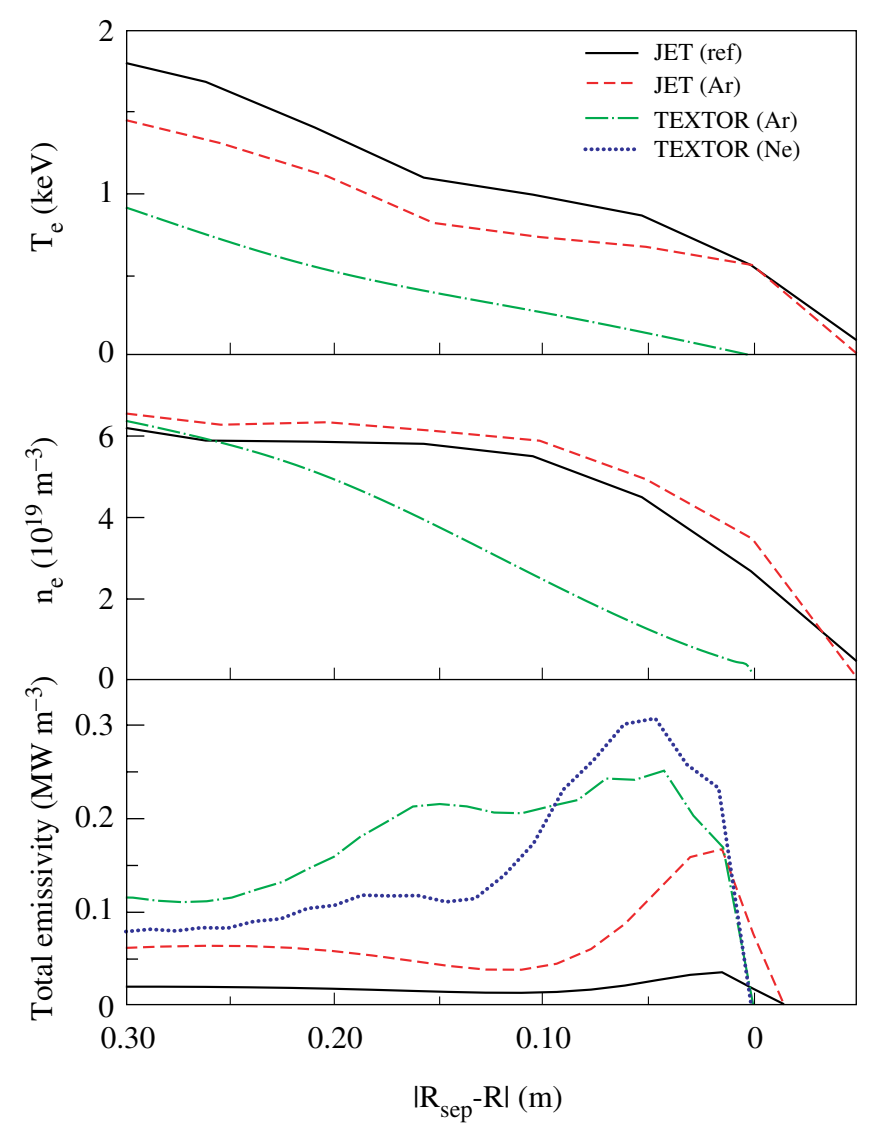

Figure 3. The electron temperature, density and Abel inverted radiation profiles overlaid with the TEXTOR profiles. Both TEXTOR and JET plasmas were near $(0.85-0.95)$ the Greenwald density limit, and heated by neutral beams to about the same heating power density. Neon or argon radiating mantles [10] create the same temperature and density profile on TEXTOR. The radius is plotted as real distance from the plasma edge to compare the JET and TEXTOR radiating mantle widths. The JET radiation profile excludes the $3 \mathrm{MW}$ of $X$-point radiation by using only bolometer chords from the upper half of the plasma.

the impurity transport is uncertain since the argon densities inferred from charge exchange recombination spectroscopy differ from those inferred from $\mathrm{x}$-ray emission.

With the impurity radiation, the ELMs became more frequent and smaller (figures 2 and 6). The energy drop per ELM was about $0.5 \%$ of the plasma energy. This magnitude in a reactor would avoid significant erosion by instantaneous ELM energy deposition. Also, core radiation and the ELMs were losing nearly all the power from the plasma (figure 6), so that the power handling in the divertor is similar to detachment between ELMs [8] since little power is conducted directly to the strike point.

The no-argon reference plasma was at the high-density, low edge electron temperature limit of the type-I ELM behaviour [9]. The argon radiation further lowered the edge electron temperature and slightly increased the edge density. The plasma electron pressure was approximately reduced about $30 \%$. JET typically observes that the global energy confinement is related to the edge pressure [9], and these radiation mantle experiments follow that trend. 


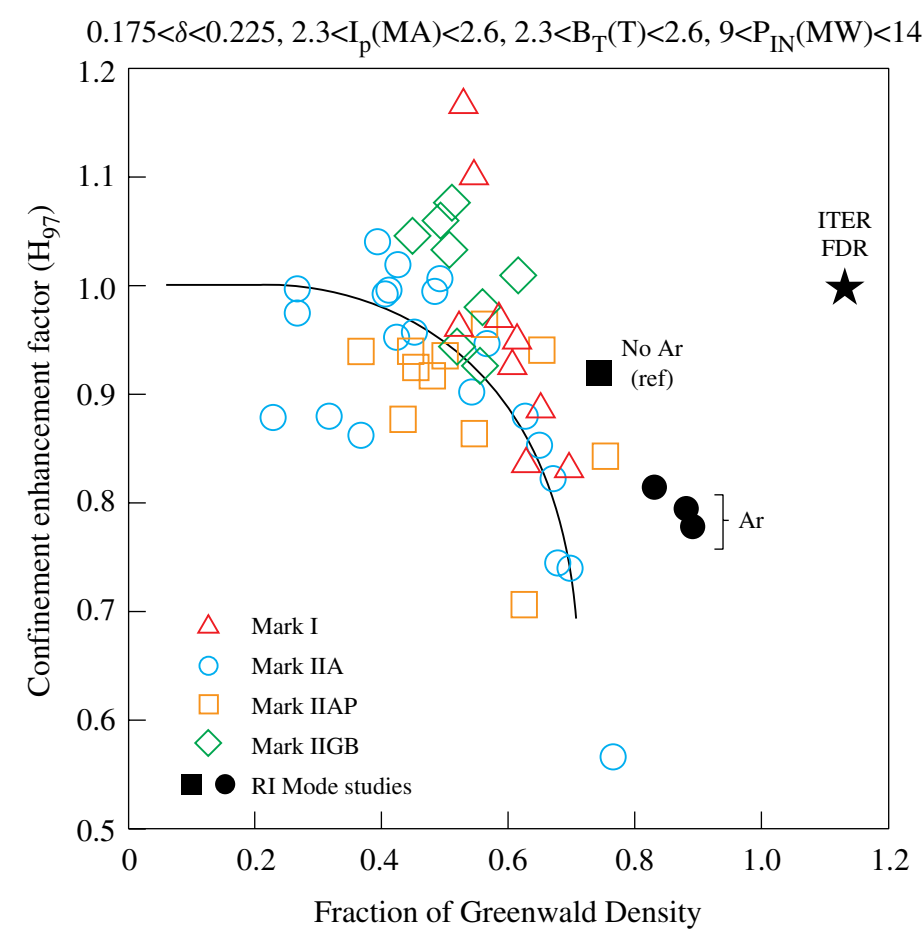

Figure 4. The normalized confinement plotted against the normalized density. The hollow data points are non-radiating JET plasmas at similar current, field, heating power, and triangularity. The argon radiation mantle and reference plasmas (solid points) are the only ones in the septum configuration. The solid curve is the JET empirical confinement degradation observed with density increases due to deuterium gas fuelling [9].

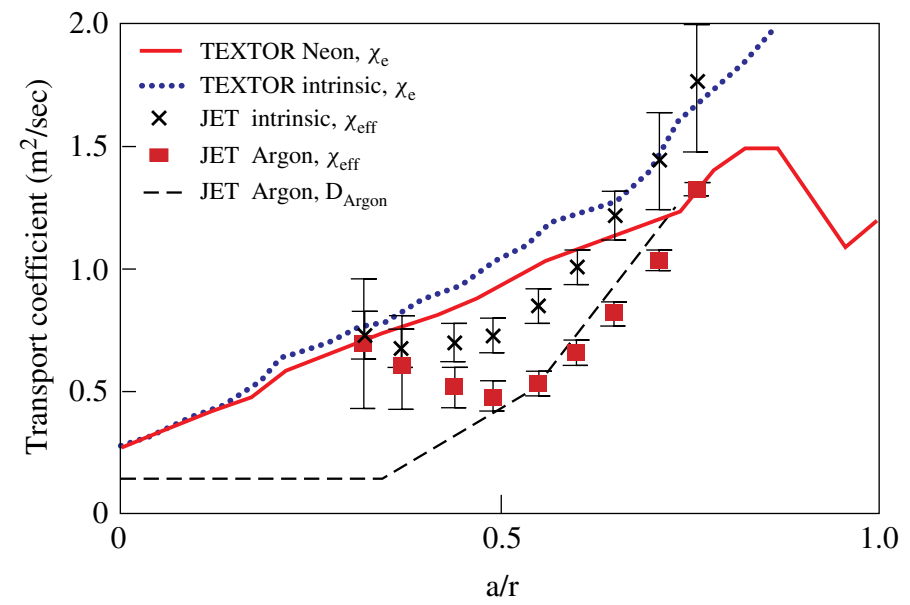

Figure 5. The profile of several transport coefficients indicates similar core confinement improvement in TEXTOR and JET. The TEXTOR electron $\chi$ is from [3]. 


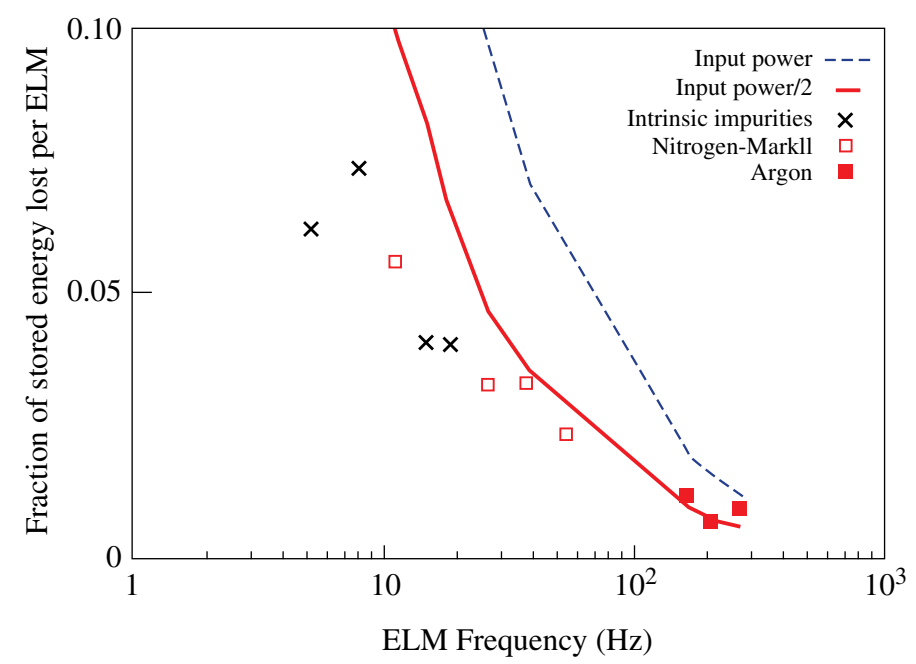

Figure 6. The fraction of total plasma energy lost at each ELM plotted as a function the observed ELM frequency. The dashed curve is the amount of energy needed to be lost at each ELM for the ELMs to transport all the input power. The solid curve is the amount of energy needed for the ELMs to transport one-half of the input power (since approximately one-half of the input power is radiated in the plasma core).

Thus the loss of confinement can be superficially understood as the typical JET energy confinement response to the reduction in the edge pressure [9]. The radiation mantle increased the ELM frequency causing more power to be lost out of the plasma volume by the ELMs. This behaviour pattern has been quantified in several JET publications by characterizing the ELM effect upon confinement $[12,13]$. However, for the case of the radiation mantles, the loss of confinement is not as large as was described by Fishpool [12], where a $200 \mathrm{~Hz}$ ELM frequency would have been accompanied by an $\mathrm{H} 89$ reduction to 1.4 instead of the 1.6 observed here. Apparently, the positive RI effect on the core conductivity (figure 5) mitigates the JET empirical ELM or edge pedestal pressure reduction in confinement, but does not eliminate it completely.

A preliminary JET experiment used argon radiation mantles to simulate the enhanced performance regimes found on ISX, DIII-D, and TEXTOR. Radiation mantles of about 50\% core radiation and $70 \%$ total radiation were achieved. The experiments yielded three interesting results:

(1) Radiative mantle plasmas were obtained with higher density (normalized to the Greenwald limit) when compared with JET plasmas at the same triangularity, field and current.

(2) The no-argon, septum configuration at high deuterium gas fuelling was the highest density, good confinement plasma in the JET data base at these conditions.

(3) The role of the edge pressure in reducing the global confinement seemed to obscure the radiation induced improvement in the core confinement.

Correlation of the JET transport coefficients with shear stabilized ITG turbulence and gyroBohm scaling has not yet been studied. Possible future experiments include:

(1) Further optimization of the impurity type, deuterium and impurity fuelling rates, and divertor configurations is warranted.

(2) Scaling of the pedestal and core transport coefficients might identify regimes where the core confinement effect can exceed the edge pedestal effect on global confinement. 
(3) Other regimes (besides the ELMy H-Mode) should be evaluated since they might become competitive with the ELMy H-Mode as a reactor regime.

\section{References}

[1] Janeschitz G et al 1996 Proc. 16th Int. Conf. on Fusion Energy (Montreal, 1996) vol 2 (Vienna: IAEA) p 755

[2] Ongena J et al 1999 Plasma Phys. Control. Fusion A 41379

[3] Messiaen A et al 1996 Nucl. Fusion 3639

[4] Lazarus E A et al 1985 Nucl. Fusion 25135

[5] Jackson G L et al 1999 J. Nucl. Mater. 266-269 380

[6] Gruber O et al 1995 Phys. Rev. Lett. 744217

[7] Kallenbach A et al 1996 Plasma Phys. Control. Fusion 382097

[8] Matthews G L et al 1999 Nucl. Fusion 3919

[9] Saibene G et al 1999 Nucl. Fusion 391591

[10] Unterberg B et al 1997 Plasma Phys. Control. Fusion 39 B189

[11] Mast K F and Krause H 1985 Rev. Sci. Instrum. 56969

[12] Fishpool G 1998 Nucl. Fusion 381373

[13] Zhang W, Tubbing B J D and Ward D J 1998 Plasma Phys. Control. Fusion 40335 